\title{
Application of molecular typing to the epidemiology of Streptococcus pneumoniae
}

Lucinda M C Hall

\begin{abstract}
The spread of antibiotic resistance and the development of new vaccines have focused attention on the epidemiology of Streptococcus pneumoniae over recent years. While serotyping and the determination of antibiotic resistance remain primary methods for characterising pneumococci, molecular typing can add greater discrimination and complementary information. Methods based on restriction fragment length polymorphism within total DNA or non-specific polymerase chain reaction provide information representative of the whole genome and can be used to recognise closely related isolates from different sources, whether in the investigation of possible cross infection at the local level or in the investigation of national or international spread of antibiotic resistant strains. Fingerprinting of penicillin binding protein genes adds further information in the analysis of penicillin resistant isolates. The use of a combination of typing methods to analyse both the genome as a whole and specific loci has led to the realisation that pneumococci undergo horizontal gene transfer much more often than most other bacterial species. In particular the spread of penicillin resistance has been characterised by a combination of the spread of epidemic strains, transfer of chromosomal resistance genes from such strains into other genetic backgrounds, and transfer of capsule genes resulting in the switch of serotypes within strains. In the future molecular typing will have an important role in discovering whether widespread vaccination leads to genetic modification of the pneumococcal population causing invasive disease.

(f Clin Pathol 1998;51:270-274)
\end{abstract}

Keywords: Streptococcus pneumoniae; molecular typing; epidemiology

Department of Medical Microbiology, St Bartholomew's and the Royal London School of Medicine and Dentistry, Turner Street, London E1 2AD, UK; email: 1.m.c.hall@mds. qmw.ac.uk L M C Hall

Correspondence to: Dr Hall.

Accepted for publication 30 January 1998

Streptococcus pneumoniae is a human pathogen of major importance causing pneumonia, bacteraemia, and bacterial meningitis with high rates of mortality and long term morbidity, as well as less serious infections such as otitis media and sinusitis. Until recent years the organism was invariably susceptible to penicillin, although even appropriate treatment was not always successful. However, the arrival and rapid spread of penicillin resistance, often concomitant with resistance to other antimicrobials, has dramatically altered our perception of the threat this organism poses. The focus of attention is now turning towards disease prevention, with re-examination of the role and potential impact of more widespread vaccination. Currently available vaccines cover 23 of the 90 known serotypes (that is, polysaccharide capsule types), representing about $90 \%$ of the organisms responsible for invasive disease. These vaccines are based on the capsular polysaccharide and are not immunogenic in young children, one of the groups at highest risk. Conjugate vaccines with the polysaccharide linked to a protein moiety, analogous to the Hib vaccine for Haemophilus influenzae type $\mathrm{b}$, are undergoing clinical trials at the present time - these overcome the problems of immunogenicity but for technical reasons can only cover up to 11 serotypes.

Epidemiological markers provide a means of distinguishing different subgroups within the species and hence of addressing specific questions about the epidemiology of pneumococcal disease. In general such questions can be divided into two categories: are particular subgroups of the species responsible for different clinical manifestations of infection? And, how does the organism spread within human populations? However, inextricably linked with such questions is the need to understand the population structure and evolution of Str pneumoniae. The use of antibiotics has already selected for changes in the genetic make up of this species-future widespread vaccination programmes will also represent a significant evolutionary pressure that might bring about further modifications over a relatively short time scale. In this review I shall describe the various typing methods applied to pneumococci, assessing their value and limitations. I shall then describe recent findings revealed by molecular typing methods, particularly relating to the spread of penicillin resistance in this species, and consider future directions for molecular epidemiology.

\section{Pneumococcal serotype}

Pneumococci have long been subdivided by

consensus usage has for some time been the Danish system, with all typing sera provided by the Statens Serum Institut, Copenhagen. ${ }^{1}$ Serotyping is based on the structural diversity of the capsular polysaccharide. The first stage of serotyping involves the use of pools of sera, and then individual sera from the positive pool, to assign a number designation. For some of these numbers, such as 1,3 , and 14 , no further subtyping is possible and hence the number is referred to as a serotype. For others, such as 6 , 
19 , and 23, subtyping sera can distinguish further subtypes such as $6 \mathrm{~A}$ and $6 \mathrm{~B}, 19 \mathrm{~F}$ (First), $19 \mathrm{~A}, 19 \mathrm{~B}$ and 19C, and so on; in these cases the number (for example, 6) is referred to as the serogroup, and the number plus letter (for example, 6A) as the serotype or subtype. This nomenclature reflects the cross reactivity of subtypes within a serogroup. ${ }^{12}$

Encapsulation is a prerequisite for virulence in pneumococci, allowing the organism to evade phagocytic cells. However, in the presence of anticapsular antibody, complement activation and phagocytosis can proceedhence the protection provided by vaccines composed of capsular polysaccharide. Because immunity is specific to the polysaccharide structures presented (whether by vaccination or natural infection), the determination of capsule type - that is, serotyping - is and will remain the primary typing method for pneumococci. Molecular genetic methods based on polymorphism of capsule genes are being investigated as an alternative to classical serotyping but will provide an indirect assessment of the polysaccharide actually being produced.

\section{Antibiotic susceptibility profile}

The determination of antimicrobial susceptibilities is of major clinical significance in informing treatment decisions but can also be considered as a typing method. Antimicrobial resistance in pneumococci is not plasmid mediated as in most other species, but involves either modification of chromosomal genesoften by horizontal gene transfer (penicillin and probably sulphonamide and trimethoprim resistance $)^{3-5}$ - or chromosomally located transposons (tetracycline, kanamycin, erythromycin, and chloramphenicol resistance). ${ }^{6} \mathrm{Re}-$ sistance profiles dependent on mobile DNA do not make ideal epidemiological markers because of their potential instability, and the discrimination afforded is limited. Nevertheless, as for serotyping, the biological and clinical significance of antimicrobial resistance accounts for its importance in the characterisation of pneumococci.

\section{Multilocus enzyme electrophoresis}

Multilocus enzyme electrophoresis (MLEE) has been used both as an epidemiological typing method and to investigate population structure in pneumococci. ${ }^{7-14}$ The method involves separating soluble enzymes by nondenaturing electrophoresis (usually in starch gels), staining for activity, and scoring polymorphism in migration rate for the same enzyme in different isolates. ${ }^{15}$ Typically at least 10 "housekeeping" enzymes are assayed, and the results-expressed as the proportion of enzymes for which two isolates have the same electrophoretic variant-can be used as a measure of genetic relatedness. The underlying assumptions are that electrophoretic variants arise from neutral mutations in the encoding gene, that the enzymes assayed derive from loci representative of the genome as a whole, and that each locus is independent of the others. Isolates indistinguishable by MLEE can be considered to belong to the same clone, in other words to have a recent common ancestor. MLEE results are frequently presented as a dendrogram of genetic relatedness. It should be noted that such a dendrogram does not necessarily correlate with the ancestry of the current isolates, since two isolates may share the same allele either because they derive from a common ancestor, or because one of them has acquired the allele by recombination (horizontal gene transfer). ${ }^{16}$ Several lines of evidence suggest that recombination is rather common in pneumococci, highlighting the importance of the above proviso. ${ }^{3101718}$

MLEE profiles make excellent epidemiological markers and have been used extensively in tracing the spread of penicillin resistance in pneumococci. However, they are cumbersome to perform, and for rapid and discriminatory typing (although not for population structure analysis) MLEE is tending to be replaced by pulsed field gel electophoresis or other methods described below.

\section{Restriction fragment length polymorphism and non-specific PCR} A survey of recent publications on the epidemiology of pneumococci shows that comparison of restriction fragments separated by pulsed field gel electrophoresis (PFGE) or field inversion gel electrophoresis is probably the most commonly used molecular typing method at present. ${ }^{13}{ }^{19-25}$ This involves digesting total genomic DNA with a restriction enzyme that has few sites (usually SmaI or ApaI), and separating the resulting large fragments (typically $50-200 \mathrm{~kb}$ ) in a fluctuating electric field. Two isolates producing the same pattern of bands can, as for MLEE, be considered to belong to the same strain or clone. The interpretation of patterns sharing some but not all bands is more difficult. Tenover et al have suggested guidelines for interpretation of PFGE patterns in point source outbreaks ${ }^{26}$; these depend on the ability to identify an isolate representing the index case and thus are not fully applicable to the context of longer term spread over wider geographical distances. Under the Tenover guidelines, isolates that differ in up to three bands from the index pattern can be considered highly related-as this many changes could arise from a single point mutation in a restriction site - and isolates differing in up to six bands are possibly related. However, if an index pattern cannot be identified, isolates with a larger number of band differences between them are not necessarily unrelated since they may each have diverged by a smaller amount from a common ancestor. A working rule that has proved useful in several contexts in our laboratory is that isolates can be considered to belong to a cluster of related isolates if they differ by not more than three bands from the most similar member of the cluster - in practice such clusters can be recognised readily by eye.

Other methods based on restriction fragment length polymorphism (RFLP) have also been used for pneumococci. These include restriction fragment end labelling followed by analysis of fragments of a few hundred base 
pairs in length, ${ }^{27}$ and ribotyping, based on detection of restriction fragments containing ribosomal RNA genes. ${ }^{828-30}$ Hermans et al have compared the discrimination of these three RFLP typing methods and found a close correlation between PFGE and end labelling, with ribotyping being less discriminatory. ${ }^{27}$ In this study ribotyping was performed with only a $16 \mathrm{~S}$ probe; it is possible that discrimination would be increased if both $16 \mathrm{~S}$ and $23 \mathrm{~S}$ had been used together, as in other investigations.

The same group has also applied PCR techniques to fingerprinting pneumococci. ${ }^{27} 31$ Primers were based either on an enterobacterial repetitive sequence (ERIC), or on pneumococcal repetitive sequences (BOX). Again discrimination with these PCR methods was similar to that found with PFGE. However, PCR fingerprinting methods need to be rigorously controlled and may have low interlaboratory reproducibility.

Applied and interpreted with intelligence, any of the above methods can be used to recognise highly related pneumococcal isolates and to distinguish between unrelated isolates that may share the same serotype. They should not, given currently available information, be used to quantitate degrees of relatedness or infer ancestry based on the numbers of shared bands. (This is because these methods, as used for typing, do not meet the criteria required for genetic markers in the context of population genetics-restriction fragments or unknown PCR products that differ between isolates cannot be considered as homologous alleles of the same locus, nor is variation in different restriction fragments independent (a single restriction site change will affect fragments on both sides of it). ${ }^{32}$

\section{Penicillin binding protein (PBP) profiles} and PBP gene (pbp) fingerprints

Much of the recent interest in the epidemiology of pneumococci involves tracing the spread of penicillin resistance, which in pneumococci is entirely due to altered penicillin binding proteins. Methods are therefore required to distinguish between the acquisition of new modifications and the spread of existing ones. Modified PBPs are classically analysed by detecting bound radiolabelled benzylpenicillin on SDS-PAGE separated proteins: polymorphisms in the PBPs are detected as differences in the migration and intensity of bands after autoradiography/fluorography. ${ }^{8} 1112$ Sequence analysis of the three PBP genes characterised to date-pbp2b, pbp2x, and pbp1a-revealed that modifications result from recombination with homologous genes from a different source, thought to be oral streptococci, forming mosaic genes. ${ }^{33}$ RFLP analysis of these genes (often referred to as fingerprinting) following PCR amplification proves to be a sensitive method of detecting different variants. In early publications restriction fragments were radiolabelled, separated on a DNA sequencing gel, and detected by autoradiography. ${ }^{717}$ However, adequate resolution can also be obtained on smaller acrylamide or agarose gels in which fragments are detected by staining ${ }^{18} 20$ (and Hall L, unpublished data).

\section{Summary of the information that typing} methods can provide

The various typing methods described above differ in the kind of information they provide. Serotyping reveals the type of capsule being produced, but since the serotype is determined by a set of genes clustered together in a cassette structure, it only gives information about one segment of the genome. MLEE, RFLP, and PCR based analyses are believed to reflect characters scattered throughout the genome, and hence give a picture of the overall genetic background of an isolate. PBP analysis and PBP gene fingerprinting again relate to specific loci-their main interest is obviously in the investigation of penicillin resistant isolates. Using a combination of complementary methods it is therefore possible to derive independent information on the genetic background of an isolate, its capsule locus, and its PBP loci; such analyses have revealed some interesting and unexpected findings as discussed below.

What typing has told us about the epidemiology of pneumococcal disease

Investigations of serotype prevalence in various collections of isolates have led to some important general observations ${ }^{233-35}$ : the relative prevalence of serotypes differs between carriage isolates and those from invasive disease; there are wide differences in the age distribution of different serotypes; the prevalence of different serotypes shows geographical variation; and the majority of penicillin resistance (and multiple antimicrobial resistance) occurs in a restricted range of serotypes, all of which are common in children. Molecular typing (including MLEE) has added to this general picture by showing that most serotypes include genetically diverse organisms, and do not appear to constitute distinct subsets or clones. ${ }^{1011} 14$ This is in contrast to the situation with Haemophilus influenzae in which type b organisms do comprise a clone that is responsible for the majority of invasive disease. ${ }^{36}$

However, it is in the investigation of spread of penicillin resistance that molecular typing has been employed most intensively. Such studies have sought to determine the relative importance of clonal dissemination of resistant strains, dissemination of resistance determinants to new strains, and independent acquisition of new resistance determinants. A recent publication by Hermans et al exemplifies many of the points made in other studies. ${ }^{18}$ The study involved all penicillin resistant isolates from 39 Dutch hospitals from March 1995 to March 1996, covering the majority of the Dutch population. Eighty nine resistant isolates $(25 \%$ with high and $75 \%$ with intermediate level resistance) were recovered. The genotype or genetic background of the isolates was assessed by restriction fragment end labelling (RFEL), and revealed 21 unique isolates, while 68 isolates clustered into nine groups, of which the two largest contained 22 and 18 isolates. Isolates from the largest cluster were invariably 
multiresistant, although the profile of resistance varied. Comparison to isolates from other sources showed that this genotype was indistinguishable from a multiresistant strain of serotype $23 \mathrm{~F}$ that was first described in Spain and has since spread to many parts of the world. The Dutch cluster included 11 isolates of serogroup 19 as well as 11 of serogroup 23. Examination of PBP genes revealed one isolate with a pattern that differed from the others. The second cluster represented isolates with a genotype indistinguishable from a multiresistant strain of serotype $9 \mathrm{~V}$, also believed to have originated in Spain and spread widely, at least in Europe. This cluster included isolates of serotype 14 as well as serogroup 9 , with a range of resistance profiles, and again one isolate with a variant PBP gene pattern.

These findings are typical of those described in other studies. ${ }^{8}{ }^{911-13} 19-222529{ }^{37}$ First, there is a small number of multiresistant strains that appear to have spread internationally, as well as others with more local spread. Second, collections of resistant isolates always include various apparently genetically unrelated isolates. Third, within the common strains there is evidence of horizontal transfer of genes, including genes involved in antimicrobial resistance and those involved in serotype determination. It is further likely that at least some of the unrelated isolates have acquired their resistance determinants from the common strains: Hermans' study showed some isolates with PBP genes indistinguishable from the major clusters, but it should also be noted that recombination may not involve entire genes so that new restriction profile types could be produced by this mechanism. These points illustrate the complementary nature of the various typing methods that can be employed in Str pneumoniae: serotyping and antibiogram and/or PBP typing would hide the extent of spread of particular strains, since both depend on genes that are not necessarily representative of the genetic background of the isolate; genotyping alone would fail to demonstrate the biologically and clinically relevant characters of serotype and antimicrobial resistance.

The Dutch study further illustrates the value of good epidemiological data in combination with typing. Where a number of indistinguishable isolates had been submitted from the same laboratory, it was possible to look at potential routes of transmission. There appeared to have been four instances where an organism was spread to other patients in the same hospital room, and in one case a patient transferred between hospitals was implicated in starting a new outbreak. All the patients involved in this case had chronic obstructive pulmonary disease and pneumococci were isolated from their sputum, so it is likely that they were long term carriers. It was not reported that any of the patients suffered invasive pneumococcal disease. Evidence for the transmission of multiresistant strains between carriers and as a cause of invasive infection in hospitals and other institutions has also been reported. ${ }^{38-44}$ Penicillin resistant pneumococci may well come to be considered as important nosocomial pathogens despite their conventional label as community acquired organisms.

\section{Future perspectives in the epidemiology of Str pneumoniae}

Over the next 10 years conjugate vaccines are likely to be used widely in intensive vaccination programmes. Will this result in the replacement of serotypes included in the vaccine with equally virulent strains carrying different or even novel types of capsule ${ }^{45}$ The evidence to date shows that pneumococci have the genetic capacity to switch serotype, and transfer of virulence genes between strains is also undoubtedly possible. Molecular typing will have an important role in monitoring the impact of widespread vaccination on the characteristics of pneumococci causing disease.

Other developments over the next few years will include exploitation of the complete sequence of the pneumococcal genome which is likely to be released to the public domain in 1998. Together with advances in technology such as high density oligonucleotide arrays (DNA chips), this will allow typing methods to target large numbers of specific genes. The detection of polymorphism in virulence genes and correlation to different manifestations of pneumococcal infection promises to be a powerful tool for molecular epidemiology in the future.

1 Lund E, Henrichsen J. Laboratory diagnosis, serology and epidemiology of Streptococcus pneumoniae. In: Bergan T, Norris JR, eds. Methods in microbiology, vol 12. New York: Norris JR, eds. Methods in micr

2 Robbins JB, Austrian R, Lee C-J, et al. Considerations for formulating the second-generation pneumococcal capsular polysaccharide vaccine with emphasis on the cross-reactive types within groups. F Infect Dis 1983;148:1136-59.

3 Dowson CG, Hutchison A, Brannigan JA, et al. Horizontal transfer of penicillin binding protein genes in penicillin resistant clinical isolates of Streptococcus pneumoniae. Proc Natl Acad Sci USA 1989;86:8842-6.

4 Adrian PV, Klugman KP. Mutations in the dihydrofolate reductase gene of trimethoprim-resistant isolates of Streptococcus pneumoniae. Antimicrob Agents Chemother 1997; 41:2406-13.

5 Maskell JP, Sefton AM, Hall LMC. Mechanism of sulfonamide resistance in clinical isolates of Streptococcus pneumoniae. Antimicrob Agents Chemother 1997;41:2121p. 6.

6 Klugman KP. Pneumococcal resistance to antibiotics. Clin Microbiol Rev 1990;3:171-96.

7 Munoz R, Coffey TJ, Daniels M, et al. Intercontinental spread of a multiresistant clone of serotype $23 \mathrm{~F}$ Streptococcus pneumoniae. F Infect Dis 1991;164:302-6.

8 McDougal LK, Facklam R, Reeves M, et al. Analysis of multiply antimicrobial-resistant isolates of Streptococcus pneumoniae from the United States. Antimicrob Agents Chemother 1992;36:2176-84.

9 Versalovic J, Kapur V, Mason EO, et al. Penicillin-resistant Streptococcus pneumoniae strains recovered in Houston: identification and molecular characterization of multiple clones. F Infect Dis 1993;167:850-6.

10 Hall LMC, Whiley RA, Duke B, et al. Genetic relatedness within and between serotypes of Streptococcus pneumoniae from the UK: analysis of multilocus enzyme electrophoresis, pulsed field gel electrophoresis, and electrophoresis, pulsed field gel electrophoresis, and
antimicrobial resistance patterns. f Clin Microbiol 1996;34: 853-9.

11 Sibold C, Wang J, Henrichsen J, et al. Genetic relationships of penicillin-susceptible and -resistant Streptococcus pneumoniae strains isolated on different continents. Infect Immun 1992;60:4119-26.

12 Reichmann P, Varon E, Gunther E, et al. Penicillin-resistant Streptococcus pneumoniae in Germany: genetic relation-
ship to clones from other European countries. $\mathcal{F ~ M e d}$ Microbiol 1995;43:377-85.

13 McDougal LK, Rasheed JK, Biddle JW, et al. Identification of multiple clones of extended-spectrum cephalosporinresistant Streptococcus pneumoniae isolates in the United States. Antimicrob Agents Chemother 1995;39:2282-8.

14 Takala AK, Vuopio-Varkila J, Tarkka E, et al. Subtyping of common pediatric pneumococcal serotypes from invasive disease. F Infect Dis 1996;173:128-35. 
15 Selander RK, Caugant DA, Ochman H, et al. Methods of multilocus enzyme electrophoresis for bacterial population
genetics and systematics. Appl Environ Microbiol 1986;51: genetics

16 Maynard Smith J, Smith NH, O'Rourke M, et al. How clonal are bacteria? Proc Natl Acad Sci USA 1993;90:43848.

17 Coffey TJ, Dowson CG, Daniels M, et al. Horizontal transfer of multiple penicillin-binding protein genes, and capsular biosynthetic genes, in natural populations of Streptococcus pneumoniae. Mol Microbiol 1991;5:2255-60

18 Hermans PWM, Sluijter M, Elzenaar K, et al. Penicillinresistant Streptococcus pneumoniae in the Netherlands: results of a 1-year molecular epidemiologic survey. F Infect Dis 1997;175:1413-22.

19 Doit C, Denamur E, Picard B, et al. Mechanisms of the spread of penicillin resistance in Streptococcus pneumoniae strains causing meningitis in children in France. $\mathcal{f}$ Infect Dis 1996;174:520-8.

20 Moissenet D, Valcin M, Marchand V, et al. Molecular epidemiology of Streptococcus pneumoniae with decreased epidemiology of Streptococcus pneumoniae with decreased
susceptibility to penicillin in a Paris children's hospital. $f$ susceptibility to penicillin in a Par

21 Barnes DM, Whittier S, Gilligan PH, et al. Transmission of multidrug-resistant serotype $23 \mathrm{~F}$ Streptococcus pneumoniae in group day care. evidence suggesting capsular transformation of the resistant strain in vivo. F Infect Dis 1995;171:890-6.

22 Figueiredo AM, Austrian R, Urbaskova P, et al. Novel penicillin-resistant clones of Streptococcus pneumoniae in the Czech Republic and in Slovakia. Microb Drug Res 1995 1:71-8.

23 Moreno F, Crisp C, Jorgensen JH, et al. The clinical and molecular epidemiology of bacteremias at a university hospital caused by pneumococci not susceptible to penicillin. $f$ Infect Dis 1995;172:427-32.

24 Gasc AM, Geslin P, Sicard AM. Relatedness of penicillinresistant Streptococcus pneumoniae serotype 9 strains resistant Streptococcus pneumoniae serotype 9 strain

25 Vaz Pato MV, Belo de Carvalho C, Tomasz A, the Multicenter Study Group. Antibiotic susceptibility of Streptococcus pneumoniae isolates in Portugal. A multicenter study between 1989 and 1993. Microb Drug Res 1995;1:59-69.

26 Tenover FC, Arbeit RD, Goering RV, et al. Interpreting chromosomal DNA restriction patterns produced by pulsed-field gel electrophoresis: criteria for bacterial strain typing. f Clin Microbiol 1995;33:2233-9.

27 Hermans PWM, Sluijter M, Hoogenboezem T, et al. Comparative study of five different DNA fingerprint techniques for molecular typing of Streptococcus pneumoniae strains. $\mathcal{f}$ Clin Microbiol 1995;33:1606-12.

28 Harakeh H, Bosley GS, Keihlbauch JA, et al. Heterogeneity of rRNA gene restriction patterns of multiresistant serotype 6B Streptococcus pneumoniae strains. 7 Clin Microbiol 1994;32:3046-8.
29 Kell CM, Jordens JZ, Daniels M, et al. Molecular epidemiology of penicillin-resistant pneumococci isolated in ology of penicillin-resistant pneumococci is
Nairobi, Kenya. Infect Immun 1993;61:4382-91.

30 Urwin G, Yuan MF, Hall LMC, et al. Pneumococcal meningitis in the North East Thames Region UK: epidemiology and molecular analysis of results. Epidemiol Infect 1996; 117:95-102

31 van Belkum A, Sluijter M, de Groot R, et al. Novel BOX repeat PCR assay for high-resolution typing of Streptococcus pneumoniae strains. F Clin Microbiol 1996;34:1176-9.

32 Swofford DL, Olsen GJ, Waddell PJ, et al. Phylogenetic inference. In: Hillis DM, Moritz C, Mable BK, eds. Molecular systematics. Sunderland, MA: Sinauer Associates, 1996:407-514.

33 Coffey TJ, Dowson CG, Daniels M, et al. Genetics and molecular biology of $\beta$-lactam-resistant pneumococci. Microb Drug Res 1995;1:29-34.

34 Scott JAG, Hall AJ, Dagan R, et al. Serogroup-specific epidemiology of Streptococcus pneumoniae: associations with age, sex, and geography in 7000 episodes of invasive disease. Clin Infect Dis 1996;22:973-81.

35 Tomasz A. Antibiotic resistance in Streptococcus pneumoniae. Clin Infect Dis 1997;24(suppl 1:S85-8.

36 Musser JM, Kroll JS, Moxon ER, et al. Clonal population structure of encapsulated Haemophilus influenzae. Infect Immun 1988;56:1837-45.

37 Coffey TJ, Berron S, Daniels M, et al. Multiply antibioticresistant Streptococcus pneumoniae recovered from Spanish hospitals (1988-1994): novel major clones of serotypes 14, 19F and 15F. Microbiology 1996;142:2747-57.

38 Raymond J, Bingen E, Brahimi N, et al. Pneumococcal meningitis resistant to penicillin and nosocomial transmission in pediatric hospitals confirmed by genomic analysis. Arch Pediatr 1996;3:1239-42.

39 Gould FK, Magee JG, Ingham HR. A hospital outbreak of antibiotic-resistant Streptococcus pneumoniae. F Infect 1987;15:77-9.

40 Mandigers CMPW, Diepersloot RJA, Dessens M, et al. A hospital outbreak of penicillin-resistant pneumococci in the hospital outbreak of penicillin-resistant pn

41 Millar MR, Brown NM, Tobin GW, et al. Outbreak of infection with penicillin-resistant Streptococcus pneumoniae in a hospital for the elderly. F Hosp Infect 1994;27:99-104

42 Moore EP, Williams EW. Hospital transmission of multiply antibiotic-resistant Streptococcus pneumoniae. F Infect 1988;16:199-200.

43 Reichler MR, Rakovsky J, Slacikova M, et al. Spread of multidrug-resistant Streptococcus pneumoniae among hospitalised children in Slovakia. f Infect Dis 1996;173: 374-9.

44 Reichler MR, Allphin AA, Breiman RF, et al. The spread of multiply resistant Streptococcus pneumoniae at a day care centre in Ohio. F Infect Dis 1992;166:1346-53.

45 Lipsitch $M$. Vaccination against colonizing bacteria with multiple serotypes. Proc Natl Acad Sci USA 1997;94:6571- 